\title{
Vídeos, Questões e Desempenho: uma análise quantitativa em cursos de engenharia
}

\author{
Alberto Bastos do Canto Filho - alberto.canto@ufrgs.br - PPGIE - UFRGS \\ José Valdeni de Lima - valdeni@inf.ufrgs.br - PPGIE - UFRGS \\ Carla Schwengber ten Caten - tencaten@producao.ufrgs.br - Escola de Engenharia - \\ UFRGS \\ Liane Margarida Rockenbach Tarouco - liane@penta.ufrgs.br - PPGIE - UFRGS
}

Resumo: A contribuição deste artigo é uma análise quantitativa de resultados obtidos numa metodologia em que são utilizados vídeos de curta duração e questões complexas como complemento a aulas expositivas tradicionais. Foi realizada uma investigação envolvendo 210 estudantes de diversos cursos de engenharia divididos em dois grupos: o grupo de controle com aulas tradicionais e o grupo experimental, que teve acesso a conteúdo complementar constituído por vídeos de curta duração produzidos por estudantes e questões complexas. A avaliação quantitativa realizada mostrou que os estudantes pertencentes ao grupo experimental tiveram um desempenho melhor do que o grupo de controle.

Palavras Chave - vídeo, motivação, aprendizagem baseada em problema

Abstract: The contribution of this paper is a quantitative analysis of results obtained according to a methodology in which short videos and evaluation questions are used as a complement to conventional lectures. An investigation involving 210 students from various engineering courses was conducted. The students were divided into two groups: the control group with traditional lectures and the experimental group. The experimental group had access to additional content (short videos produced by students and evaluation questions). The quantitative analysis of students' performance shows that the experimental group performed better than the control group.

Keywords - video, motivation, problem based learning

\section{Introdução}

O termo “Apagão da Engenharia” tem sido utilizado para expressar o receio de que o crescimento econômico do país venha a ser comprometido devido à falta de engenheiros (CANTO e LODER, 2010). Este risco pode ser minimizado de duas formas: 1) Através da ampliação da oferta de vagas em cursos de engenharia. 2) Através da redução da evasão dos estudantes.

Através do programa de ampliação de vagas no ensino superior (REUNI) promovido pelo Ministério de Educação e Cultura, a escola de Engenharia da Universidade Federal do Rio Grande do Sul (UFRGS) criou novos cursos e ampliou o número de vagas em cursos existentes. Como resultado deste processo de ampliação de vagas, entre os anos de 2007 e 2010 houve um aumento de 54\% no número de ingressantes em cursos cujas disciplinas são oferecidas pelo Departamento de Engenharia Elétrica (CANTO et al., 2011). Este aumento de vagas contribuiu para o ingresso de um grupo de estudantes de não ingressariam na condição anterior, aumentando substancialmente o número de estudantes com dificuldades para 0 
acompanhamento no curso, o que resultou em aumento das taxas de retenção. Em outras palavras, os elevados investimentos realizados para o aumento de vagas para ingressantes em cursos de engenharia poderão ter um resultado inexpressivo em termos de concluintes, devido ao incremento da evasão.

A melhoria dos processos de ensino e aprendizagem é uma alternativa de mais baixo custo, capaz de aumentar o número de concluintes sem reduzir os padrões de qualidade. Ainda assim, a adoção de processos de ensino e aprendizagem mais eficazes demanda recursos adicionais nem sempre disponíveis. Por exemplo, métodos de atividade ativa geralmente são mais eficazes do que o sistema tradicional de aulas expositivas, mas demandam um maior número de horas docentes e turmas menores, o que pode implicar a necessidade de contratação de novos professores e a inviabilidade econômica.

Neste artigo é apresentada uma alternativa de aprendizagem baseada em problemas (PBL), que faz uso de vídeos de curta duração produzidos pelos próprios estudantes e de questões complexas apresentadas no Ambiente Virtual de Aprendizagem Moodle.

\section{Trabalhos Relacionados}

Ausubel, Novak, \& Hanesian (1980) sustentam que a aprendizagem por recepção é o método mais eficiente ${ }^{1}$ de aquisição do conhecimento. Ressaltam, no entanto, que a aprendizagem significativa por recepção emerge nos estágios mais avançados de maturidade cognitiva. Infelizmente, mesmo se tratando de cursos superiores não é possível assumir que todos os estudantes se encontrem nos níveis mais avançados de maturidade cognitiva; consequentemente, uma parte dos estudantes não está preparada para os métodos de ensino e aprendizagem comunmente utilizados em cursos de engenharia.

A Maturidade Cognitiva avançada possui duas componentes essenciais, que são o estado de prontidão (motivação) e a disponibilidade de conceitos subsunçores em quantidade e qualidade adequada (AUSUBEL, NOVAK e HANESIAN, 1980; CANTO e SUSIN, 2006). Estes dois fatores se situam em domínios diferentes em termos da taxonomia de Bloom (BLOOM e KRATHWOHL, 1956), e necessitariam objetivos específicos apropriados: enquanto a motivação se situa no domínio das atitudes (domínio afetivo), a disponibilidade de subsunçores se situa no domínio do conhecimento (domínio cognitivo).

A Teoria da Autodeterminação (GAGNÉ e DECI, 2005; RYAN e DECI, 2000; VANSTEENKISTE, LENS e DECI, 2006) tem sido utilizada para trabalhar a componente motivacional da maturidade, relacionando o estilo de regulação e a motivação. A pior situação é a falta de motivação situação em que o objeto de estudos não é valorizado e não há qualquer tipo de fato que motive a ação. A seguir, vem a regulação externa situação em que o estudante se percebe pressionado a realizar uma atividade para conseguir um prêmio ou livrar-se de um castigo. Este tipo de regulação é associado aos métodos de aprendizagem baseados em estímulo-resposta (SKINNER, 2005) e ao aprendizado mecânico. Dentro da categoria "motivação extrínseca" existem ainda os estilos de regulação Introjetada (motivação fundamentada em questões tais como culpa, orgulho ou ansiedade), Através de Identificação (há uma valorização

\footnotetext{
${ }^{1}$ Definições Baseadas na NBR 9241-11:

Eficiência é a relação entre o resultado alcançado e os recursos utilizados.

Eficácia é a extensão na qual as atividades planejadas são realizadas e os resultados planejados alcançados.
} 
consciente da ação), Integrada (os objetivos estão associados aos interesses pessoais). Este último estilo de controle resulta numa motivação extrínseca tão boa quanto a motivação intrínseca (os objetivos são interesses pessoais).

Estudos realizados no contexto desta investigação (CANTO, FERREIRA e BERCHT, 2012) mostram que frequentemente o baixo desempenho dos estudantes está mais relacionado à falta de motivação do que a questões cognitivas. Nestes estudos constatou-se que, na inexistência de motivação intrínseca, a motivação “por nota” pode ser utilizada como forma de incentivo para que os estudantes se engajem nas atividades propostas.

A metodologia apresentada neste artigo propõe que se explore a motivação por nota como uma alternativa para desenvolver uma atitude reflexiva entre os estudantes para os quais os métodos tradicionais não são suficientemente motivadores.

Na seção a seguir será apresentada a metodologia proposta.

\section{Metodologia}

A Aprendizagem Significativa é necessariamente uma aprendizagem ativa, pois o estabelecimento de relações substantivas e não arbitrárias entre as novas proposições e a estrutura cognitiva prévia (conceitos subsunçores) é resultante de uma atividade cognitiva que envolve processos tais como a comparação, a identificação de similaridades e diferenças, resolução das diferenças e modificação da estrutura cognitiva por meio de integração ou diferenciação (AUSUBEL, NOVAK e HANESIAN, 1980). Os métodos de aprendizagem por recepção poderão ser caracterizados como métodos de aprendizagem ativa quando o material exposto tiver um significado lógico para o estudante e a aula expositiva for suficientemente motivadora para que se realizem estas operações cognitivas. Frequentemente os métodos de aprendizagem por descoberta são chamados de “aprendizagem ativa”, o que geralmente é verdade, pois a descoberta normalmente demanda atividade cognitiva.

A metodologia de Ensino e Aprendizagem proposta neste artigo tem como objetivo apresentar um complemento às aulas tradicionais, visando atingir um grupo de estudantes que, embora disponha das condições cognitivas necessárias para o aprendizado significativo, deixa de realizar as operações cognitivas necessárias para tal devido à falta de motivação ou por hábitos de estudos baseados em aprendizagem mecânica. Caracterizada como um método de Aprendizagem Baseada em Problemas, a proposta apresentada fundamenta-se na apresentação de vídeos de curta duração e questões que avaliam continuadamente se os Objetivos Educacionais foram alcançados.

Nas subseções a seguir serão apresentadas as etapas desta metodologia.

\subsection{Definição dos Objetivos Educacionais}

A clara definição dos objetivos educacionais é pré-requisito necessário para o desenvolvimento do sistema de avaliação. Sugere-se utilizar a Taxonomia Revisada de Bloom (KRATHWOHL, 2002), que considera duas dimensões:

Conhecimento - subdividida nas seguintes categorias: 1 - Conhecimento factual; 2 - Conhecimento do Conceito; 3 - Conhecimento de Métodos Relacionados; 4

- Metaconhecimento.

Processo - subdividida nas seguintes categorias: 1- Lembrar; 2 - Entender; 3 Aplicar; 4 - Analisar; 5 - Avaliar; 6 - Criar. 
Observe que a categoria mais simples de processo (Lembrar) é uma categoria de aprendizagem mecânica, enquanto os processos mais complexos (Entender, Aplicar, Analisar, Avaliar, Criar) exigem o aprendizado significativo.

Para que se avalie se um determinado objetivo educacional foi alcançado, é necessário que se desenvolva um sistema de avaliação compatível, caso contrário os resultados apurados não representarão a realidade que se deseja mensurar.

\subsection{Elaboração de vídeos de curta duração}

O vídeo é uma alternativa de aprendizagem por recepção que pode ser utilizada como complemento às expositivas tradicionais. O Quadro 3-1 apresenta uma comparação entre aulas expositivas tradicionais e o uso de vídeos. Observe que existem diversas funcionalidades inerentes ao uso desta tecnologia, que complementam os pontos fracos das aulas expositivas tracionais.

\begin{tabular}{|c|c|}
\hline Aulas Expositivas Tradicionais & Vídeos \\
\hline Ensino Presencial & Ensino a Distância \\
\hline Hora, Local e Duração Fixos & $\begin{array}{l}\text { A qualquer hora, em qualquer lugar, } \\
\text { quanto tempo desejar }\end{array}$ \\
\hline Roteiro Adaptável conforme as dúvidas & Roteiro Fixo \\
\hline $\begin{array}{l}\text { Reapresentação de conceitos: } \\
\text { Necessita que o estudante solicite ao } \\
\text { docente, se expondo frente à turma } \\
\text { Representa perda de tempo para uma } \\
\text { parcela da turma }\end{array}$ & $\begin{array}{l}\text { Reapresentação de conteúdos: } \\
\text { Tantas vezes quantas quiser } \\
\text { Não demanda tempo de outros } \\
\text { estudantes }\end{array}$ \\
\hline $\begin{array}{l}\text { Permite alguma interatividade, desde que } \\
\text { não tome um tempo excessivo. }\end{array}$ & $\begin{array}{l}\text { Interatividade limitada à navegação } \\
\text { (voltar, pausar, rever). }\end{array}$ \\
\hline Número limitado de estudantes & $\begin{array}{l}\text { Número de estudantes virtualmente } \\
\text { infinito. }\end{array}$ \\
\hline $\begin{array}{l}\text { Lógica de Custo Variável: os custos } \\
\text { docentes e de espaço físico são } \\
\text { proporcionais ao número de estudantes }\end{array}$ & $\begin{array}{l}\text { Lógica de Investimento: Gastos } \\
\text { antecipados na produção do vídeo. }\end{array}$ \\
\hline Realidade ao vivo. & $\begin{array}{l}\text { Representação da realidade (possui perdas } \\
\text { dependendo da resolução, frames por } \\
\text { segundo, qualidade do áudio, etc.) }\end{array}$ \\
\hline $\begin{array}{l}\text { Proporciona o convívio natural com } \\
\text { colegas de turma. }\end{array}$ & $\begin{array}{l}\text { Acesso individual; Necessita definição de } \\
\text { alternativas complementares para o } \\
\text { convívio com colegas. }\end{array}$ \\
\hline
\end{tabular}

Quadro 3-1. Aulas expositivas tradicionais X Vídeos

\subsection{Desenvolvimento de Questões}

Nesta etapa, devem-se desenvolver questões que avaliam se o objetivo educacional foi alcançado. Enfatiza-se aqui a necessidade de que as questões sejam concebidas de uma forma coerente com o objetivo educacional. Por exemplo, considerando que o nível de complexidade de complexidade do objetivo seja Entendimento de um conceito, o uso de questões que avaliam se conceito foi memorizado ou se o estudante é capaz de aplicar o conceito somente serviria para avaliar se o estudante situa-se aquém ou além do objetivo estabelecido. 


\subsection{Aplicação}

Nesta etapa os vídeos e questões são disponibilizados para os estudantes através de Ambiente Virtual de Aprendizagem (AVA). Os estudantes devem ser informados sobre os prazos e o peso que a resolução das questões terá no sistema de avaliação da disciplina. A utilização de questões para avaliar se os estudantes alcançaram os objetivos educacionais caracteriza esta metodologia como uma Aprendizagem Baseada em Problemas, e tem objetivo afetar os estudantes que, por falta de motivação ou por hábitos de estudo, não realizam sistematicamente as reflexões necessárias sobre os conceitos abordados. O sistema de avaliação continuada induzirá o estudante a refletir sobre os conceitos abordados, proporcionado a aprendizagem significativa. Estes novos conceitos estarão consolidados antes da próxima aula, na qual poderão servir como âncora para as novas proposições apresentadas.

A seguir será apresentado experimento realizado com o objetivo avaliar quantitativamente a eficácia desta metodologia.

\section{Aplicação Experimental da Metodologia}

A metodologia aqui proposta foi aplicada em caráter experimental na disciplina de "Eletricidade", ministrada para os cursos de Engenharia Civil, Engenharia Química, Engenharia de Alimentos, Engenharia de Materiais, Engenharia Metalúrgica, Engenharia de Produção da Universidade Federal do Rio Grande do Sul. Esta avaliação ocorreu no segundo semestre de 2014; neste período a disciplina tinha 210 estudantes matriculados em quatro turmas.

Foram considerados seguintes objetivos educacionais:

Objetivo 1: capacitar os estudantes a analisar circuitos utilizando as Leis de Kirchoff;

Objetivo 2: capacitar os estudantes analisar circuitos de corrente alternada;

Foram utilizados vídeos de curta duração produzidos por estudantes de engenharia através da iniciativa "Me Salva":

Vídeos sobre Leis de Kirchoff: Lei dos nós e Lei das Malhas

Vídeos sobre Corrente Alternada: Introdução à CA e Capacitores.

Utilizou-se o Moodle para disponibilizar os vídeos e questões para os estudantes. Foram utilizados os seguintes recursos:

Atividade Scorm: Tela de boas-vindas (Figura 4-1), que explica a dinâmica dos trabalhos para os estudantes. Rotinas Javascript utilizaram a interface Scorm para leitura do Nome do Estudante, do número de identificação e atribuição de notas. A atribuição de notas foi utilizada como um artifício de navegação que explora as funcionalidades de sequenciamento do Moodle em função da nota obtida em atividades já realizadas. Foi atribuída a nota 9,9 para estudantes com cartão ímpar e a nota 9,7 para estudantes com cartão par; Na configuração dos questionários (vide item Questionários, a seguir) especificou-se que somente os estudantes com nota entre 9,6 e 9,8 poderiam visualizar as questões sobre Leis de Kirchoff e que somente os estudantes com notas entre 9,8 e 10 poderiam acessar o questionário sobre Corrente Alternada. Isto é, esta atividade separou os estudantes em dois grupos:

o Grupo LK: estudantes de cartão par, que tiveram acesso aos vídeos e questões sobre Leis de Kirchoff (e não tiveram acesso aos vídeos e questões sobre Corrente Alternada); 
o Grupo CA: estudantes de cartão ímpar, que tiveram acesso aos vídeos e questões sobre Corrente Alternada (e não tiveram acesso aos vídeos e questões sobre Leis de Kirchoff);

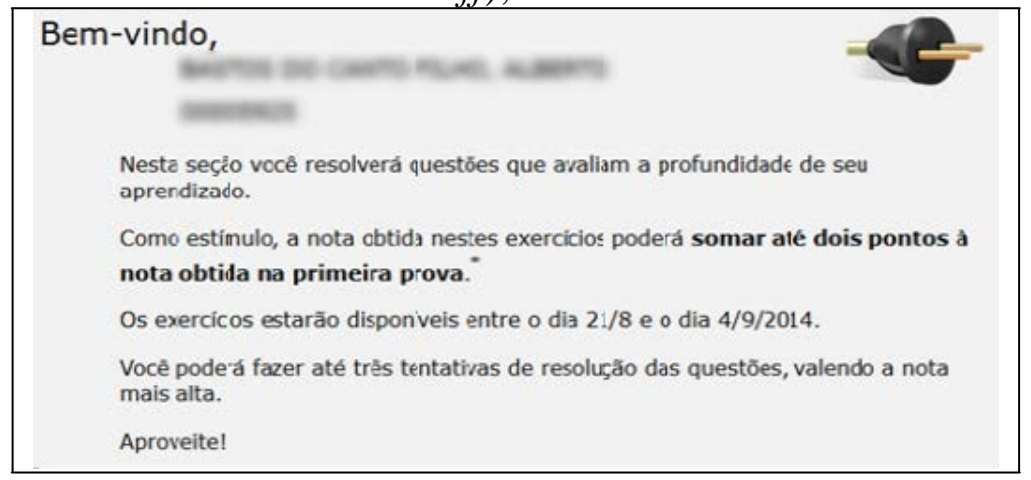

Figura 4-1. Telas de boas-vindas

Questionários: Foram disponibilizados dois questionários que utilizaram o recurso "Questão Calculada" do Moodle:

o Questionário sobre Leis de Kirchoff: questionário contendo seis questões que avaliam a capacitação do estudante para analisar circuitos utilizando as leis de Kirchoff. Foram utilizadas 5 questões calculadas, cada uma das quais com 20 variantes de resposta. Este questionário foi disponibilizado apenas para os estudantes do grupo LK (estudantes com cartão par). A Figura 4-2 mostra como o artifício de atribuição de notas para a atividade de boas vindas foi configurado no Moodle para restringir o acesso; a figura mostra também que o acesso ao vídeo foi fornecido através de um link no corpo do questionário.

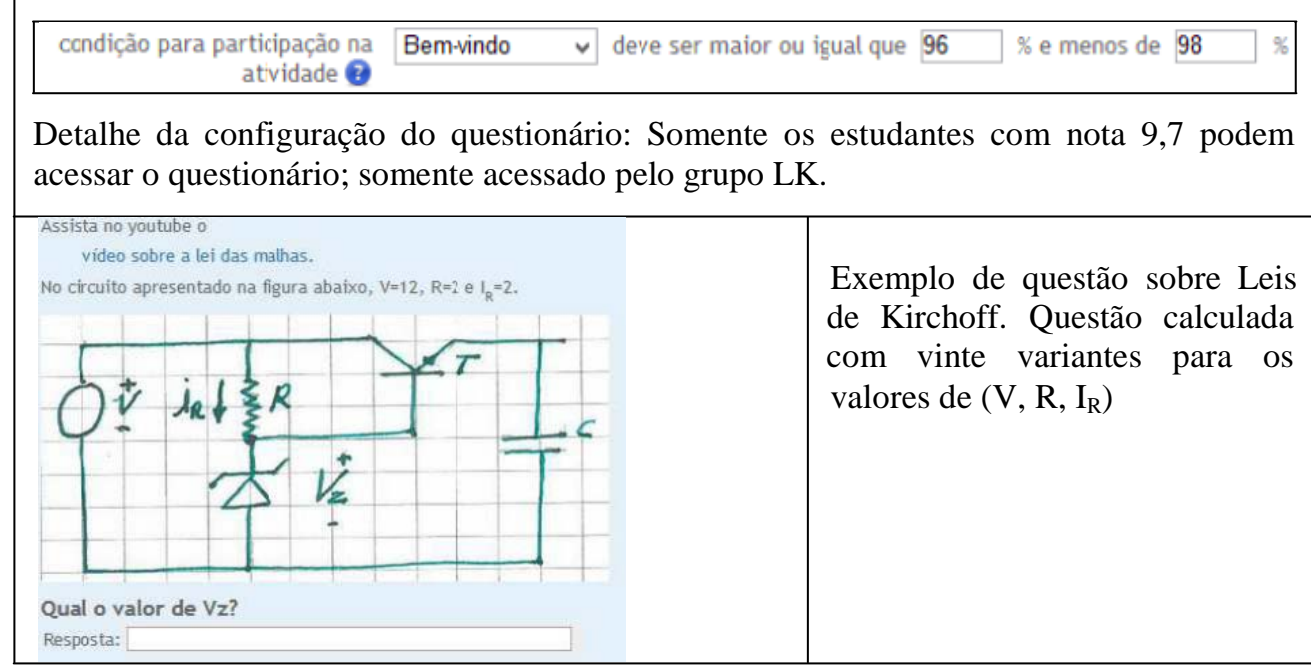

Figura 4-2. Questionário sobre as Leis de Kirchoff

o Questionário sobre Corrente Alternada: questionário com oito questões que avaliam a capacidade do estudante de resolver problemas sobre corrente alternada. Foram utilizadas questões calculadas com quarenta variantes de respostas.

Foi dado um prazo de duas semanas ara que os estudantes realizassem as atividades do Moodle; Os estudantes foram informados que os escores obtidos seriam convertidos em "pontos extras”, somados à nota da prova. 
A prova presencial apresentou duas questões elaboradas especificamente com o objetivo de avaliar a proporção em que os objetivos educacionais foram alcançados.

Na seção a seguir serão apresentados os resultados obtidos.

\section{Resultados}

Os resultados apresentados nesta seção comparam o desempenho dos estudantes que aprenderam no sistema tradicional (aulas expositivas) com o desempenho dos estudantes que utilizaram a metodologia proposta (aulas expositivas + vídeos de curta duração + sistema da avaliação continuada). Os resultados consideram os dois objetivos educacionais e os dois grupos de estudantes descritos na seção acima (Quadro 5-1).

\begin{tabular}{|l|c|c|}
\hline & $\begin{array}{c}\text { Grupo } \\
\text { Experimental }\end{array}$ & $\begin{array}{c}\text { Grupo de } \\
\text { Controle }\end{array}$ \\
\hline $\begin{array}{l}\text { Objetivo 1: capacitar os estudantes a analisar } \\
\text { circuitos utilizando as Leis de Kirchoff; }\end{array}$ & LK & CA \\
\hline $\begin{array}{l}\text { Objetivo 2: capacitar os estudantes analisar } \\
\text { circuitos de corrente alternada; }\end{array}$ & CA & LK \\
\hline
\end{tabular}

LK: Grupo dos estudantes que teve acesso aos vídeos e questões sobre leis de Kirchoff

CA: Grupo dos estudantes que teve acesso aos vídeos e questões sobre Corrente Alternada

\section{Quadro 5-1. Análise de Resultados conforme Objetivo Educacional}

A Tabela 1 mostra as notas médias obtidas na questão da prova sobre as leis de Kirchoff. Observe que os 106 estudantes do grupo LK (que teve acesso aos vídeos e questões sobre leis de Kirchoff) tiveram uma nota média maior $(5,25)$ do que a média dos estudantes do grupo CA (4.43).

Tabela 1- Desempenho na questão sobre Leis de Kirchoff

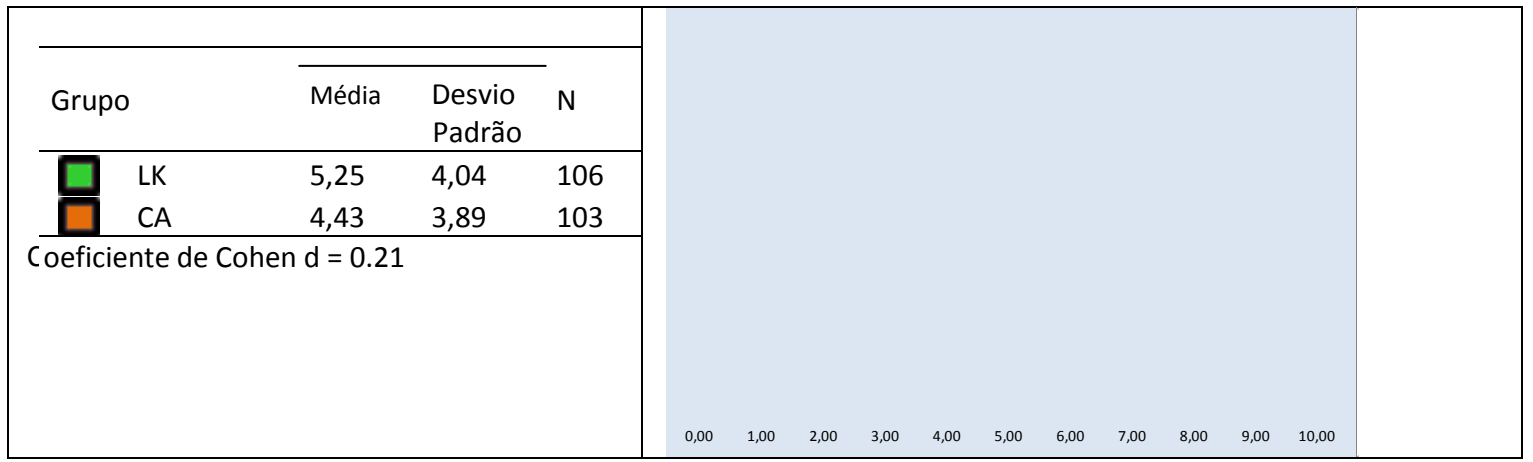

A Tabela 2 mostra as notas médias obtidas na questão da prova sobre corrente alternada.

Observe que os 103 estudantes do grupo CA (que teve acesso aos vídeos e questões sobre Corrente Alternada) tiveram uma nota média maior $(6,47)$ do que os estudantes do grupo LK $(3,77)$. 
Tabela 2- Desempenho na questão sobre Corrente Alternada

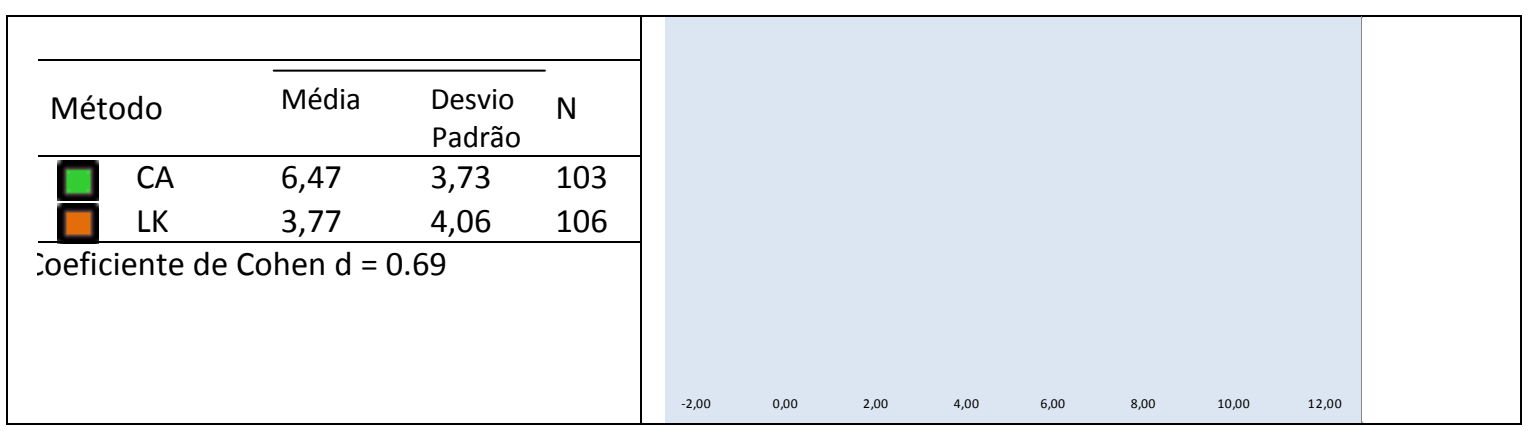

Houve significativa diferença entre os resultados obtidos nas duas situações avaliadas: para o objetivo relacionado à aprendizagem de Leis de Kirchoff apurou-se um coeficiente de Cohen $\mathrm{d}=0.21$, enquanto o para o objetivo relacionado à aprendizagem de conceitos de Corrente Alternada o coeficiente obteve-se um coeficiente de Cohen $\mathrm{d}=0.69$.

Estes resultados evidenciam que o uso de vídeos de curta duração associados ao uso de questões complexas avaliadoras possuem potencial melhoria dos processos de ensino e aprendizagem tradicional, mas que os resultados são influenciados por outros fatores, entre eles:

Conhecimento prévio do conceito: o impacto da metodologia é inversamente proporcional ao domínio prévio do conceito abordado. O tema "Leis de Kirchoff” é abordado em disciplinas que precedem ao ensino de eletricidade, enquanto o tema "Corrente Alternada” é visto pela primeira vez, o que contribuiu para a diferença de impacto observada.

Didática dos vídeos de curta duração: à medida que os vídeos de curta duração apresentem os conceitos com uma didática diferente daquela que é utilizada nas aulas expositivas, eles poderão favorecer ou prejudicar o processo de aprendizagem.

Características do sistema de avaliação: os resultados apurados nas provas podem variar substancialmente dependendo das questões resolvidas. Por exemplo, a utilização de uma questão similar a um exemplo apresentado em aula expositiva poderá aproximar os resultados obtidos pelos dois grupos (grupo de controle e grupo experimental). Por outro lado, o uso de questões similares àquelas apresentadas no material complementar pode afastar os resultados médios obtidos pelos dois grupos.

Outros fatores: a aprendizagem é um processo dependente de inúmeros fatores relacionados aos hábitos e atitudes dos estudantes. Por exemplo, o uso de outros materiais buscados autonomamente ou o estudo em grupo podem ser fatores que mascaram os resultados quantitativos obtidos.

A investigação relatada neste trabalho mostra que o complemento de aulas expositivas com vídeos de curta duração associado à avaliação continuada é uma metodologia capaz de melhorar o desempenho médio dos estudantes. Atribui-se esta melhoria a fatores motivacionais, didáticos e cognitivos.

A atribuição de nota aos estudantes é um fator de motivação extrínseca para a realização das tarefas propostas. A forma como este estímulo é percebido pode ser diferente para cada estudante, enquadrando-se em qualquer uma das classificações de estilo regulatório que geram motivação extrínseca:

No melhor caso, a nota é percebida pelo estudante como uma expressão do seu valor pessoal e de sua competência (controle através de identificação). 
No pior caso, a nota é percebida como um prêmio ou castigo imposto externamente (regulação externa).

A questão a ser debatida relaciona-se ao resultado alcançado pelos estudantes que percebem a atribuição de nota como um fator de regulação externa. Tipicamente, estes estudantes realizarão a tarefa buscando conseguir "o prêmio" com o mínimo de dispêndio de energia. Embora esta condição não seja desejada, deve-se ponderar que:

Caso este estímulo não fosse oferecido, o estudante não realizaria qualquer esforço cognitivo para o aprendizado;

O “esforço mínimo para obtenção da nota” poderá ser um esforço cognitivo suficiente para o aprendizado significativo, desde que a resolução das questões assim o exija.

Há estudos (RICHARDSON, 2005) que consideram o tipo de abordagem como uma característica individual; o estudante pode preferir utilizar a abordagem superficial, a abordagem profunda ou uma abordagem estratégica, que adota a abordagem superficial se possível, mas adota a abordagem profunda sempre que necessário para obtenção do grau necessário.

Outras alternativas motivacionais, tais como personalizações que configuram os materiais apresentados de acordo com as motivações individuais podem possuir um maior potencial motivacional, mas exigem demandam mais recursos (custos).

Finalmente, deve-se considerar que dificilmente a abordagem quantitativa aborda todas as variáveis relevantes em processos complexos que envolvem os seres humanos, cultura e comportamento, havendo a necessidade de desenvolvimento de investigações qualitativas complementares.

\section{Agradecimentos}

Agradecemos aos professores Valdirene Verdum e Fioravante Brum, responsáveis pela disciplina "Eletricidade"; Agradecemos aos diretores e integrantes da iniciativa "Me Salva" pela produção e disponibilidade dos vídeos utilizados, Miguel Andorffy, André Corletta e Rafael Ortiz.

\section{Referências Bibliográficas}

AUSUBEL, D. P.;; NOVAK, J. D. e HANESIAN, H. Psicologia educacional. Rio de Janeiro: Interamericana, 1980.

BLOOM, B. S. e KRATHWOHL, D. R. Taxonomy of Educational Objectives: The Classification of Educational Goals. Handbook I: Cognitive Domain. New York: Longmans, 1956.

CANTO, A. B. Do et al. Desafios da educação em engenharia: vocação, formação, exercício profissional, experiências metodológicas e proposições. In: OLIVEIRA, V. F.; CHAMBERLAIN, Z. M. (Eds.). 1. ed. Brasília: ABENGE, 2011. .

CANTO, A. B. Do;; FERREIRA, L. e BERCHT, M. Objetos de aprendizagem no apoio à aprendizagem de engenharia: explorando a motivação extrínseca. RENOTE- Revista Novas Tecnologias na Educação, v. 10, n. 3, 2012. 
CANTO, A. B. Do e LODER, L. L. Engenheiros - quantidade x qualidade. In: XXXVIII CONGRESSO BRASILEIRO DE EDUCAÇÃO EM ENGENHARIA. Anais... Fortaleza - CE: [s.n.], 2010.

CANTO, A. B. Do e SUSIN, A. A. Liderança situacional aplicada ao ensino. In: ANAIS DO XXXIV COBENGE. Anais... Passo Fundo: [s.n.], 2006.

GAGNÉ, M. e DECI, E. L. Self-determination theory and work motivation. Journal of Organizational behavior, v. 26, n. 4, p. 331-362, 2005.

KRATHWOHL, D. R. A revision of Bloom's taxonomy: An overview. Theory into practice, v. 41, n. 4, p. 212-218, 2002.

RICHARDSON, J. T. E. Students’ Approaches to Learning and Teachers’ Approaches to Teaching in Higher Education. Educational Psychology, v. 25, n. 6, p. 673-680, doi:10.1080/01443410500344720, 2005.

RYAN, R. M. e DECI, E. L. Self-determination theory and the facilitation of intrinsic motivation, social development, and well-being. American psychologist, v. 55, n. 1, p. 68, 2000.

SKINNER, B. Science and human behavior. The BF Skinner Foundation, 2005.

VANSTEENKISTE, M.;; LENS, W. e DECI, E. L. Intrinsic Versus Extrinsic Goal Contents in Self-Determination Theory: Another Look at the Quality of Academic Motivation. Educational Psychologist, v. 41, n. 1, p. 19-31, doi:10.1207/s15326985ep4101_4, 2006. 